\title{
Fault Diagnosis of Gear Based on the Variable Fuzzy Similarity Nearness
}

\author{
$\mathrm{Di} \mathrm{Wu}^{*}, 1$, Jin $\mathrm{Yao}^{2}$ and Hua $\mathrm{Li}^{2}$ \\ ${ }^{I}$ School of Energy and Power Engineering, Xihua University, Chengdu, 610065, China \\ ${ }^{2}$ School of Manufacturing Science and Engineering, Sichuan University, Chengdu, 610065, China
}

\begin{abstract}
In order to improve the validity and accuracy of fault diagnosis on mechanical equipment, this study applies fuzzy nearness approach by adjusting the traditional formulations. Besides, it points out the disadvantages in fault diagnosis of traditional nearness approach based on experimental data of gear transmission. A fuzzy nearness functionbased model is constructed to execute the fault diagnosis with the assistance of fuzzy nearness principles and fuzzy mathematical membership degree. Following that, a experimental data-based numerical example is implemented, which demonstrates that the adjusted fuzzy nearness approach performs better than the traditional one in diagnosing gear fault effectively and meeting the practical requirements of gear transmission fault diagnosis.
\end{abstract}

Keywords: Fuzzy mathematics, gear fault diagnosis, nearness degree.

\section{INTRODUCTION}

Gearbox is a speed-varied transmission mechanism, which is commonly used in changing rotation speed and transmission power for its inherent characteristics of fixed transmission ratio, large transmission torque, compact structure, high transmission efficiency and long service time etc. $[1,2]$. Gearbox is an important component of machinery, however, it is very susceptible to damaged for its complicated structure and harsh working environment. For example, invalid gear transmission is a common fault for gearbox. Gear faults are mainly constituted of gear surface damage, including gear surface pitting, abrasion, scoring, plastics deformation etc., and wheel tooth break-down. It is widely known that gear fault has direct negative effects on impeding secure reliable operation, reducing production efficiency and causing casualties etc [3]. Therefore, in recent decades, it draws great attention on researches on faults diagnosis on gearbox for its significance.

The torsional-vibration assessment and gear-fault diagnosis were made by [4] in the simple gearbox-based electromechanical system. They found gearbox torsional vibrations had a obvious impact on the torque and statorcurrent signatures [5]. A new prognosis method for the gear faults was started by [6] in dc machines. Moreover, they used hidden Markov models (HMMs) to predict the future state of fault severity. A new neuro-fuzzy diagnostic system found by [7] who developed integrating three robust signal processing techniques: the continuous wavelet transform (amplitude), beta kurtosis used the overall residual signal and the phase modulation [7].

It is very important to have an early faults diagnosis on the gears before their operations. Since that the structures of

*Address correspondence to this author at the School of Energy and Power Engineering, School of Mechanical Engineering, Xihua University, Chengdu, 610065, China; Tel: +86 28 87387056; Fax: +86 28 87387056;

E-mail:wudixc@gmail.com gears are becoming more and more complicated, it is easy to have the chain reaction so that the whole system is paralyzed once some faults happens [8]. Thus, it is necessary to eliminate all faults in an early stage so that the whole system can operate in a secure and reliable environment. In previous studies, three methods were widely used to diagnose faults, including method based on mathematical modeling, method based on signal processing and artificial intelligence-based method. Besides, more researches concentrate on neural networks-based artificial intelligence method, in which a large number of fault samples are needed for neural network training before diagnosis. However, it is easy to collect samples in normal operating state but difficult to obtain fault samples. Under the circumstances, the recognition theory based on fuzzy nearness was proposed to handle the difficulty properly. The principles of the fuzzy nearness theory are to firstly make a fuzzy nearness comparison between the timely obtained fault samples to be diagnosed and the diagnosed standard fault samples. Then, the fault causes can be figured out based on the comparison so that a better fault diagnosis can be carried out.

In this study, the principles of the fuzzy nearness will be firstly introduced and some disadvantages of traditional nearness-based approaches are discussed. In addition, the fuzzy mathematics is applied to adjust the nearness, which is then used in a numerical example. It can be concluded that the model based on adjusted nearness performs well in diagnosing faults.

\section{THEORY OF FUZZY NEARNESS}

Concept of nearness: Nearness is used to measure the closeness two fuzzy sets degree through a value ranging between 0 and 1 . When the nearness approaches to 0 , it denotes that the distance between two sets are becoming greater and the relationship is sparser. Otherwise, when the nearness approaches to 1 , it denotes that the distance 
between two sets are becoming smaller and the relationship is closer. The mathematical formula is as follows:

Assume domain $U$ and mapping $n: F(\underline{U})$, in which $F(U) \rightarrow[0,1]$ satisfies the following conditions:

(1) $n(A, A)=1, n(\varphi, U)=0$

$\{$ (2) $n(A, B)=n(B, A)$

(3) $A \subseteq B \subseteq C \Rightarrow n(A, C) \leq n(A, B) \cap n(B, C)$

Thus, $n(A, B)$ denotes the nearness between $A$ and $B$.

Concept of traditional nearness: The commonly used nearness formula is as follows:

Min Max nearness can be obtained through the following Eq. (1).

$$
n(A, B)=\frac{\sum_{j=1}^{m} X_{a j} \wedge X_{b j}}{\sum_{j=1}^{m} X_{a j} \vee X_{b j}}
$$

where $W_{E E}$ denotes the eigenvalues number in the samples, $j=1,2, \cdots, m ; X_{a j}$ and $X_{b j}$ represent the eigenvalues of the fault samples to be tested $a$ and the eigenvalues of the standard fault samples $b$, respectively; $\wedge$ and $\vee$ denote the min and max operators, respectively.

Although that the traditional nearness formula can figure out the closeness of two fuzzy sets, there are some disadvantages. Occasionally, the expected conclusions cannot be drawn by calculating Eq. (1). In this study, two sets of standard fault pressure waveform data from two sets of fuel systems are taken as an example. Assume that the normal performance $A$ and Spalling $B$ follow distributions:

$$
\begin{gathered}
A=(1,1,0.427,0.274,0.556,0.423,0.705,0.631,0.237,0.846), \\
B=(-0.504,0.627,0.924,0.549,0.554,-1,-1,-0.931,0.475,0.673) .
\end{gathered}
$$

It is calculated that the nearness equals to -1.8829 so that it is impossible to make an accurate diagnosis for it exceeds the value range of the nearness. In order to avoid misdiagnosis of the traditional nearness method, this study attempts to improve the disadvantages in the traditional nearness formula based on the fundamental principles of fuzzy nearness and the membership degree in fuzzy mathematics. It can be concluded that it plays well in fault diagnosis.

\section{INTRODUCTION TO THE IMPROVED NEARNESS}

Definition of membership function: The definition of membership function is proposed as follows:

For any set $U$, there is always a member $A(x) \in[0,1], A$ denotes a fuzzy set of $U$, then the value $A(x)$ is called the membership degree of $x$ in $A$. When $x$ changes within $U$ and $A(x)$ will follow so that $A(x)$ becomes a function, which is called the membership function of $A$ when $A(x)$ is closer to 1 , which means the membership degree is higher. Otherwise, when $A(x)$ is closer to 0 , the membership degree is lower. Thus, the membership function $A(x)$ ranging $[0,1]$ is applied to reflect the $x$ membership degree in $A$.

Normalization: The selection of membership function influences the accuracy and stability of fault diagnosis on system. When collecting the false diagnosed system signals, most system have faults that direct influences on its secure and reliable operations and they represent alarm values. Thus, the collected data from the samples must be normalized, in other words, the samples have to be standardized or normalized by Eq. (2):

$$
x=\left|\left(v_{a}-v_{0}\right) /\left(v_{b}-v_{0}\right)\right|
$$

where $x$ means sample parameter's deviation degree from normal value (dimension); $v_{0}$ means the normal value; $v_{a}$ denotes the faults values collected during operations and $v_{b}$ represents a reference value, which is the eigenvalues of the diagnosed standard fault samples.

Constructing membership function: It is common that deviation values from the normal ones in gear faults diagnosis system are large or are floating besides the normal ones and the closeness between various faults and signs are different, therefore, a new membership function in Eq. (3) based on the aforementioned conditions, the wide applicability of membership functions and the inherent faults characteristics.

$$
u(x)=-4 x^{3}+6 x^{2}-x
$$

In Eq. (3), it is reflected firstly that the variation for $u(x)$ is small when $x=0$ or $x=1$, and the function $u(x)$ changes the fastest when $x=0.5$, which demonstrates that the changes are rather small when the faults signs approach the normal values or some certain faults values. In other words, $u(x)$ can be used to reflect the fluctuation characteristics of the gear faults signs, which is beneficial for recognizing the fuzzy modes. Besides, it is also shown that when $x=0$, which means that the faults signs are normal, then the membership function $u(x)=0$. Otherwise, $u(x)=1$ when $x=1$. This membership function is designed to guarantee that the faults signs will vary besides the normal values, in which $x \in[0,1]$. Thus, the faults values that are deviated from or floating besides the normal ones can be expressed through different values after being transformed by Eq. (3).

The nearness between the faults signs to be tested and the standard faults signs can be determined by calculating the fuzzy nearness. Additionally, the gear faults can be diagnosed by applying the nearness of selection principles, which present that the ones nearest to the normal ones will be selected. Thus, the nearness formula can be expressed as:

$\delta=1-\frac{1}{n} \sum_{j=0}^{n=1}[1-u(j)]^{2}$

where $n$ denotes the eigenvalues number and $u(j)$ denotes the $j_{t h}$ membership degree of eigen value. 


\section{NUMERICAL EXAMPLE}

Vibration signals can properly indicate the state and performance of gear transmission system [8]. Based on the wavelet transform, the entropy with the mutual information, a complete system presented by [9] mixing with signal processing, feature extraction, feature selection and classification approaches for gear fault diagnosis. In our research, we choose the feature values described by [9]. to practice fuzzy recognition, which are: wavelet energy features $\left(W_{E 1}, W_{E 2}, W_{E 3}\right)$, wavelet singular values $\left(W_{S 1}, W_{S 2}, W_{S 3}\right)$, wavelet energy Shannon entropy $\left(W_{E E}\right)$, wavelet singular Shannon entropy $\left(W_{S E}\right)$, wavelet energy Renyi entropy $\left(W_{E R}\right)$, wavelet singular Renyi entropy $\left(W_{S R}\right)$.

They can be represented as a set

$X\left(W_{E 1}, W_{E 2}, W_{E 3}, W_{S 1}, W_{S 2}, W_{S 3}, W_{E E}, W_{S E}, W_{E R}, W_{S R}\right)$.

Diagnostic process: The diagnostic process figure of the improved fuzzy nearness method is described as Fig. (1).

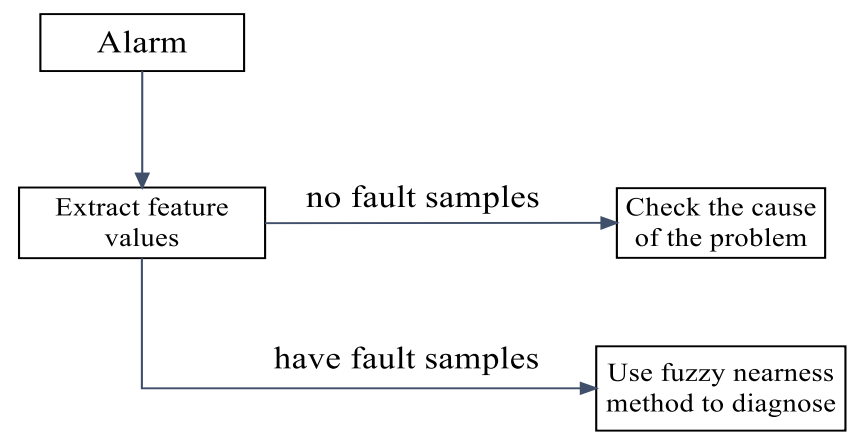

Fig. (1). Diagnostic process framework.

When there are in absence of sample fault data, technical staff check the cause of gear faults and record the fault eigenvalues. After a period of operation, we can use the fuzzy nearness method integrating fault sample data to diagnose the gear faults.

The comparison analysis by using sample data: Table 1 shows five common faults of gear system and its corresponding wavelet feature values. It can be effective for fault diagnosis by comparing the nearness between them and the collected fault symptoms.

In this study, we compare the diagnosis results between traditional nearness equation and improved nearness equation by using the following five diagnosis data samples:
Sample1: Wear

$(1,0.432,-0.896,0.483,0.234,0.272,-0.341,0.432,-0.235,0.887)$,

Sample2: Pitting

$(1,-0.569,0.231,0.856,-0.754,-0.242,0.137,0.563,-0.873,-0.573)$,

Sample3: Spalling

$(-0.429,0.561,0.871,0.746,0.472,-0.371,-1,-0.863,0.742,0.768)$,

Sample4: Broken

$(1,-0.463,0.765,0.121,-0.273,-0.398,0.431,1,-0.298,-0.336)$,

Sample5: Crack

$(0.231,0.327,-0.834,-0.311,0.836,-1,-0.133,1,0.122,-0.653)$

Nearness 1 means the closeness to the wear diagnosis, nearness 2 means the closeness to the pitting diagnosis, and so on. The diagnosis results calculated by traditional and improved nearness equation are shown in Tables $\mathbf{2}$ and $\mathbf{3}$, respectively.

As can be seen from the above results, it is not able to diagnose the actual gear fault by using traditional nearness method, which may easily get the results beyond the range of nearness and is limited to filter the data which is not undulate. However, the improved nearness model diagnoses the gear faults accurately and shows the superiority of solving this problem.

The comparison between triditional and improved nearness method in different gear fault types: In actual gear fault diagnosis, it is often to encounter the situation that needs to identify with different gear faults. We use two sets of feature values of different gear fault to identify the nearness, wear

$A=(1,0.219,-1,0.764,0.423,0.111,-0.234,0.283,-0.386,1)$, spalling

$\mathrm{B}=(-0.529,0.937,0.947,0.857,0.763,-0.856,-1,-1,0.765,0.957)$. The nearness calculated by Eq. (1) is $\delta_{A}=-6.4247$ and unable to diagnose accurately. However, the nearness calculated by Eq. (4) is $\delta_{B}=0.465281$ and can diagnose accurately.

The comparison between traditional and improved nearness method in a same gear fault type: Another condition is to identify the same gear fault with two different sample data, wear

Table 1. Faults and features extracted from its corresponding signal.

\begin{tabular}{|c|c|c|c|c|c|c|c|c|c|c|}
\hline \multirow{2}{*}{ Fault Type } & \multicolumn{10}{|c|}{ Extracted Feature Values } \\
\hline & $W_{E 1}$ & $W_{E 2}$ & $W_{E 3}$ & $W_{S 1}$ & $W_{S 2}$ & $W_{S 3}$ & $W_{E E}$ & $W_{S E}$ & $W_{E R}$ & $W_{S R}$ \\
\hline Wear & 1 & 0.219 & -1 & 0.764 & 0.423 & 0.111 & -0.234 & 0.283 & -0.386 & 1 \\
\hline Pitting & 1 & -0.632 & 0.333 & 0.463 & -0.937 & -0.754 & 0.284 & 0.388 & -1 & -0.587 \\
\hline Crack & 0.339 & 0.475 & -0.752 & -0.385 & 0.956 & -1 & -0.484 & 1 & 0.385 & -0.749 \\
\hline
\end{tabular}


Table 2. The results calculated by traditional nearness method.

\begin{tabular}{|c|c|c|c|c|c|}
\hline Samples & Nearness 1 & Nearness 2 & Nearness 3 & Nearness 4 & Nearness 5 \\
\hline \hline 1 & 8.858 & -8.514 & -1.184 & -5.794 & -8.584 \\
\hline 2 & -5.680 & 11.045 & -8.362 & 14.538 & -11.837 \\
\hline 3 & -2.053 & -8.159 & 10.479 & -2.341 & 11.935 \\
\hline 4 & -7.077 & 13.556 & -7.248 & -1.241 & -14.936 \\
\hline 5 & -1.392 & -5.150 & 2.137 & \multicolumn{2}{c|}{11.694} \\
\hline
\end{tabular}

Table 3. The results calculated by improved nearness method.

\begin{tabular}{|c|c|c|c|c|c|}
\hline Samples & Nearness 1 & Nearness 2 & Nearness 3 & Nearness 4 & Nearness 5 \\
\hline \hline 1 & 0.456292 & 0.896876 & 0.129876 & 0.429876 & 0.728376 \\
\hline 2 & 0.719386 & 0.298756 & 0.829565 & 0.110987 & 0.828936 \\
\hline 3 & 0.398375 & 0.489175 & 0.729847 & 0.209764 & 0.209764 \\
\hline 4 & 0.829487 & 0.728936 & 0.197343 & 0.624976 & 0.520974 \\
\hline 5 & 0.280937 & 0.389763 & 0.928375 & 0.324897 & 0.326733 \\
\hline
\end{tabular}

$\mathrm{A}=(1,0.219,-1,0.764,0.423,0.111,-0.234,0.283,-0.386,1)$, spalling

$\mathrm{B}=(-0.529,0.937,0.947,0.857,0.763,-0.856,-1,-1,0.765,0.957)$. The nearness results calculated by Eq.(1) and Eq.(4) are $\delta_{A}=0.659$ and $\delta_{B}=0.884$, respectively, which indicates the improved method can identify more accurately.

The validation of proposed nearness method: The improved nearness equation can not only identify the analog data but also distinguish the different types of data. We implement the proposed algorithm in Labview, and select a large number of samples to validate the algorithm. Three reprehensive sets of data of each gear fault are selected and the results are showed in Table 4. It is obvious that the diagnosis results are identical with the actual gear faults.
Thus, the improved fuzzy nearness algorithm avoid the requirement of large number of priori fault samples in neural net algorithm.

\section{CONCLUSION}

This study proposed a new fuzzy nearness-based approach, which is an improvement of the traditional fuzzy nearness method and applied it into a fault diagnosis on gear system. It can make an accurate recognition on the fuzzy modes of the collected faults samples. At the same time, it is easy to be implemented on the virtual platform Labview and presents many advantages in analyzing a large number of data. It is proven to be valuable in diagnosing practical faults.

Table 4. Sample results of improved nearness method.

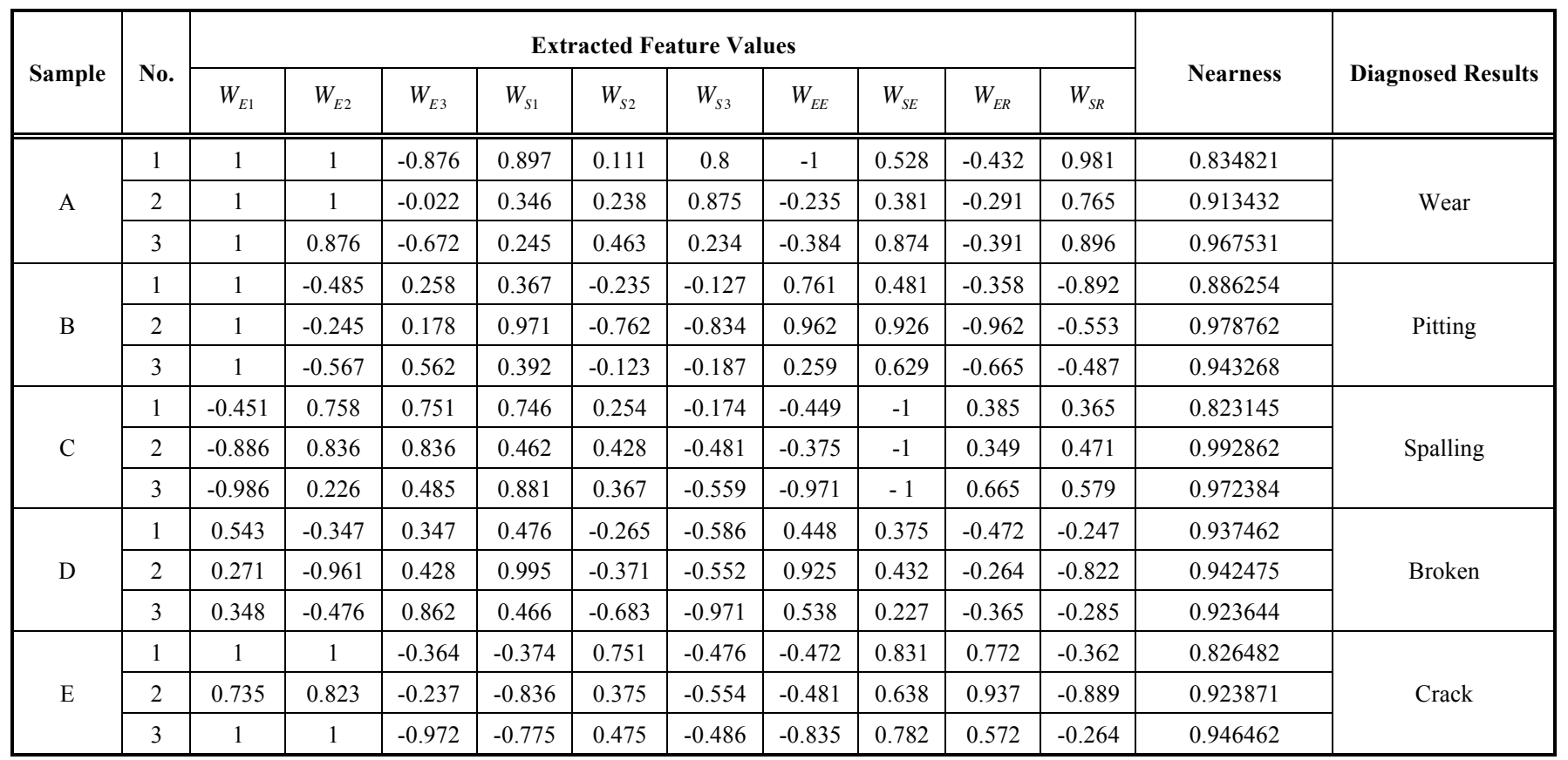




\section{CONFLICT OF INTEREST}

The authors confirm that this article content has no conflict of interest.

\section{ACKNOWLEDGEMENTS}

This research was supported by the Science \& Technology Pillar Program of Sichuan Provincial Science and Technology Department (2010GZ0173), and the Project of Research Program on Applied Fundamentals of Sichuan Provincial Science and Technology Department (2012JY0085).

\section{REFERENCES}

[1] J. S. Kang, "Continuous hidden Markov model based gear fault diagnosis and incipient fault detection", In: International Conference on Quality, Reliability, Risk, Maintenance, and Safety Engineering, 2011, pp. 486-491.

[2] X. Xu, R. P. Zhou, and Z. X. Li, "Virtual simulation analysis and experimental study on gear fault diagnosis based on wavelet neural network," In: International Conference on Machine Vision and Human-Machine Interface, 2010, pp. 55-58.
[3] M. Amarnath, and I.R.P. Krishna, "Empirical mode decomposition of acoustic signals for diagnosis of faults in gears and rolling element bearings," Science, Measurement \& Technology, IET, vol. 6, no. 4, pp. 279-287, 2012.

[4] H. Humberto, S.H. Kia, and G.A.A. Capolino, "Torsional-vibration assessment and gear-fault diagnosis in railway traction system," Industrial Electronics, vol. 58, no. 5, pp. 1707-1717, 2011.

[5] S.S.H. Zaidi, S. Aviyente, M.A. Salman, K.K. Shin, and E.G. Strangas, "Prognosis of gear failures in dc starter motors using hidden markov models," Industrial Electronics, vol. 58, no. 5, pp. 1695-1706, 2011.

[6] W.Q. Wang, F. Ismail, and F.F. Golnaraghi, "A neuro-fuzzy approach to gear system monitoring," Fuzzy Systems, vol. 12, no. 5, pp. 710-723, 2004

[7] P. Jun, G. H. Yan, X. Wang, J. Tian, and Y. G. Yang, "Gear fault detection with Wigner-Viller distribution based cepstrum approach," In: $2^{\text {nd }}$ International Conference on Computer Engineering and Technology, 2010, pp. V1-500 - V1-502.

[8] S. F. Ai, and H. Li, "Application of order cepstrum and neural network to gear fault detection," In: IMACS Multi-conference on Computational Engineering in Systems Applications, 2006, pp. 1822-1827.

[9] B. Li, P. L. Zhang, S. B. Liang, and G. Q. Ren, "Feature extraction and selection for fault diagnosis of gear using wavelet entropy and mutual information," In: $9^{\text {th }}$ International Conference on Signal Processing, 2008, pp. 2846-2850.

(C) Wu et al.; Licensee Bentham Open.

This is an open access article licensed under the terms of the (https://creativecommons.org/licenses/by/4.0/legalcode ), which permits unrestricted, non-commercial use, distribution and reproduction in any medium, provided the work is properly cited. 